\title{
Crystal Structure and Structure-Property Relationship of Halide Superionic Conductors as Solid Electrolyte for All-Solid-State Batteries Hailong Chen ${ }^{1}$ hailong.chen@me.gatech.edu
}

\begin{abstract}
All-Solid-State Batteries are a promising next-generation electrochemical energy storage technology that may replace current Li-ion batteries in the future, owing to their outstanding safety properties and high energy density. Currently its electrochemical performance and commercialization are majorly limited by the relative low performance of the solid electrolyte. Solid electrolytes with high ionic conductivity, low electronic conductivity, good chemically and elelectrochemically stability and low manufacture cost are much desired. In last two years, a group of lithium containing halides with a general formula Li3MX6 ( $\mathrm{M}=\mathrm{Y}, \mathrm{Sc}$, and $\mathrm{In}$, etc.; $\mathrm{X}=\mathrm{Br}$ or $\mathrm{Cl})$ attracted much attention as they are more all-around candidate for solid electrolyte than existing oxide and sulfide electrolytes. They are more chemical and electrochemically stable than sulfides and are much easier to synthesize. They also showed much higher ionic conductivity than that of oxides. However, their room temperature ionic conductivity is still lower than state-of-the-art sulfide electrolytes by one order of magnitude. To improve their ionic conductivity, it is critically important to understand the structure-property relationship and find the ionic conductivity governing factors in this group of compounds. Here we will share our recent research results on elucidating the crystal structure of $\mathrm{Li} 3 \mathrm{YBr} 3 \mathrm{Cl} 3$ and related compounds with using synchrotron $\mathrm{X}$-ray and neutron diffraction and pair distribution function analysis. We identified the existence of $\mathrm{Li}$ at the tetrahedral sites in the lattice, which were not reported previously. The existence of tetrahedral Li has significant impact on the energy landscape of the Li sublattice and the diffusion energy barriers in all three dimensions, which in part results in the very high room temperature ionic conductivity of $7.2 \mathrm{mS} / \mathrm{cm}$ and a very low diffusion energy barrier of $0.25 \mathrm{eV}$. The performance of all-solid-state batteries with using $\mathrm{Li} 3 \mathrm{YBr} 3 \mathrm{Cl} 3$ as the electrolyte and the design guidelines will also be discussed.
\end{abstract}

